\title{
Prosumers e redes sociais como fontes de informação mercadológica: uma análise sob a perspectiva da inteligência competitiva em empresas brasileiras ${ }^{\mathbf{1}}$
}

\author{
Prosumers and social networks as marketing information \\ sources: An analysis from the perspective of competitive \\ intelligence in Brazilian companies
}

\author{
Lidiany Kelly da Silva MARQUES² \\ Frederico VIDIGAL ${ }^{2}$
}

\section{Resumo}

\begin{abstract}
A Internet e a Web 2.0 modificaram a forma como as pessoas obtêm informação e se relacionam no meio em que vivem. É nesse contexto que as redes sociais surgem como uma fonte de informação indispensável no processo de inteligência ampla, acessível e de baixo custo, que precisa ser mais bem entendida e explorada, pois pode gerar vantagem para a empresa. Este estudo tem por objetivo verificar como as redes sociais são utilizadas na fase de coleta de informações com foco em inteligência competitiva. Procurou-se propor um modelo analítico-conceitual de verificação dessas redes sociais como fontes de informações. Para tanto, realizou-se uma pesquisa qualitativa, exploratória e descritiva, a partir de entrevistas orientadas por um roteiro semiestruturado, abarcando cinco empresas nacionais, de segmentos e portes distintos, que exercem a atividade de Inteligência Competitiva. Para a análise dos dados, elegeu-se a técnica de análise de conteúdo, e para a sistematização das informações foi utilizado o software Atlas.ti. Os resultados demonstraram que as empresas estão atentas às redes sociais como fontes de informação, pois reconhecem sua importância, principalmente pelo contato direto com os prosumers, e destacam a necessidade de sua verificação, considerando o modelo proposto como muito importante e viável para a triagem das informações mercadológicas.
\end{abstract}

Palavras-chave: Fontes de informação. Inteligência competitiva. Prosumers. Redes sociais.

\begin{abstract}
The Internet and Web 2.0 changed the way people obtain information and relate to the environment they live in. In this context, social networks emerge as indispensable sources of information on the wide intelligence process, accessible and low-cost, which need to be better understood and exploited, as they can create advantage for companies. This study aims to determine how social networks are used in the information gathering phase, focused on competitive intelligence. The intention was to propose an analytical and conceptual model of verification of social networks as sources of information. Therefore, there was a qualitative, exploratory and descriptive study based on semi-structured interviews and covering five national companies of different segments and sizes, engaged in the Competitive Intelligence activity. For the data analysis, we elected the content analysis technique, and the systematization of information was performed with the software Atlas.ti. The results showed that companies are attentive to social networks as sources of information, as they recognize their importance primarily due to direct contact with prosumers, and highlight the need for their verification, considering the model as very important and feasible for sorting market information.
\end{abstract}

Keywords: Information sources Competitive intelligence. Prosumers. Social networks.

\footnotetext{
1 Artigo elaborado a partir da dissertação de L.K.S. MARQUES, intitulada "O uso das redes sociais como fontes de inteligência competitiva: um estudo multicasos". Universidade Fumec, 2015.

2 Universidade Fumec, Faculdade de Ciências Empresariais, Programa de Mestrado em Administração da Fumec. Av. Afonso Pena, 3880, Cruzeiro, 30130-009, Belo Horizonte, MG, Brasil. Correspondência para/Correspondence to: L.K.S. MARQUES. E-mail: <marqueslidiany@gmail.com>.

Recebido em 17 de junho de 2016, versão final reapresentada em 28 de abril de 2017 e aprovado em 5 de junho de 2017.

Como citar este artigo/How to cite this article

Marques, L. K. S.; Vidigal, F. Prosumers e redes sociais como fontes de informação mercadológica: uma análise sob a perspectiva da inteligência competitiva em empresas brasileiras. Transinformação, v. 30, n. 1, p. 1-14, 2018. https://doi.org/10.1590/2318-08892018000100001
} 


\section{Introdução}

A Internet vem modificando a forma como as pessoas obtêm informação e como se relacionam no meio em que vivem, pois tornou-se um meio que permite a "comunicação de muitos com muitos" em escala global (Castells, 2003).

Uma transformação tecnológica, ocorrida na última década, modificou ainda mais a forma como a Internet promove o acesso à informação. A introdução da Web 2.0, plataforma que proporcionou um salto qualitativo nesse meio, permitiu ao usuário deixar de ser um mero espectador de notícias, para tornar-se seu produtor. Por meio de ferramentas que integram a rede global, denominadas de softwares sociais, mídias sociais ou, simplesmente, redes sociais (RS), termo que será utilizado neste trabalho, a Web 2.0 permite a criação, o compartilhamento e a alteração de todo tipo de conteúdo, de forma dinâmica e ágil, promovendo a interação por diálogo e colaboração mútua, conforme discutido por Barreto (2011).

Outra contribuição importante é a de Recuero (2009), quando afirma que as redes sociais provocaram uma série de mudanças no comportamento das pessoas, da sociedade como um todo e também das organizações, pois essas são dinâmicas e influenciadas por processos sociais como cooperação, competição e conflito.

No Brasil, segundo dados de pesquisa realizada pelo Instituto Brasileiro de Opinião Pública e Estatística (2010), 92\% dos usuários acessam a Internet em busca de informação, e 71\% dos internautas, residentes em regiões metropolitanas do país, pertencentes a distintas classes sociais, estão presentes nessas redes. A pesquisa aponta ainda que a maioria dos usuários deseja estar à frente, ou seja, busca estar entre os primeiros a saber de novidades e busca, fundamentalmente, a interação com as marcas. Os usuários não são passivos: participam, selecionam e emitem opiniões. É nesse cenário composto pela Web 2.0, redes sociais, circulação de informação e interatividade que surge o prosumer, tido como o novo consumidor, que é simultaneamente produtor e consumidor.

Para Fonseca et al. (2008), os prosumers são influenciadores do mercado, da produção ao consumo, principalmente no desenvolvimento de produtos e serviços, ao ponto, inclusive, de rejeitarem produtos que não considerem de boa qualidade. Moya (2014) afirma que nas redes sociais flui uma grande quantidade de informação, gerada pelos próprios usuários e utilizada em forma de aproveitamento e conhecimento coletivo, no sentido de melhorar qualquer aspecto da organização, inclusive sua rentabilidade.

Devido às mudanças das relações, as empresas também são afetadas e necessitam acompanhar esses avanços com a mesma velocidade. Uma contribuição importante vem da Inteligência Competitiva como técnica capaz de trabalhar a coleta de informações inteligentes de modo sistêmico e aplicado aos interesses específicos e estratégicos da organização. Trata-se de uma fundamental ferramenta para manter-se sempre à frente de seu mercado (Prescott; Miller, 2002). Para Rodrigues e Riccardi (2007), a Inteligência Competitiva auxilia na identificação de tendências no ambiente dos negócios, ou seja, permite à empresa antecipar-se a possíveis mudanças de competição ou de consumo em seu mercado e, assim, assegurar maior eficiência às estratégias adotadas. Para Tarapanoff (2001), a capacidade de monitoramento das informações sobre o ambiente e, consequentemente, a resposta aos desafios e oportunidades que são constantes no dia a dia da empresa é o que caracteriza inteligência.

No processo de monitoramento, as fontes de informação são importantes e indispensáveis; assim, Vidigal (2011) destaca que os profissionais de Inteligência Competitiva dependem de numerosas fontes secundárias de informação e que essas são mais acessíveis, devido à disponibilidade de ferramentas tecnológicas da atualidade.

Diante dessa realidade, as redes sociais vêm se tornando uma fonte de informação de suma importância e que permite às empresas acompanharem a concorrência; monitorarem o ambiente onde a organização atua; detectarem o aporte de novas tecnologias no mercado; entenderem o comportamento do consumidor e possibilitarem o aprimoramento ou desenvolvimento de novos produtos ou serviços. 
Algumas perguntas em relação ao tema destacam-se, entre elas: as redes sociais têm sido utilizadas como fontes de informações? As empresas buscam informações sobre as necessidades e expectativas dos prosumers em redes sociais? Diante das questões apresentadas, o objetivo deste artigo é verificar como o uso das redes sociais gera informações com foco em inteligência competitiva, nas empresas estudadas. Além disso, pretende-se também identificar os principais fatores pesquisados nas redes sociais que subsidiam as empresas estudadas no processo de tomada de decisão, principalmente no desenvolvimento de produtos e serviços.

\section{Internet, rede e redes sociais}

A Internet trouxe inúmeras inovações para a sociedade. Miranda (2000) afirma que uma das contribuições mais importantes foi permitir que qualquer usuário, particularmente ou institucionalmente, seja o produtor, o intermediário e o usuário de conteúdo, pois este é tudo o que é operado pela rede e possui alcance universal.

Castells $(2003$, p.7) ressalta que a Internet é a "base tecnológica para a forma organizacional da Era da Informação: a rede", apresentando-a com um conjunto de nós que estão interconectados e que tomaram um novo caminho, pois, em todos os setores da economia, desbancam as estruturas mais burocratizadas e verticalizadas, sendo altamente flexíveis e adaptáveis, características próprias de um ambiente altamente mutável.

A configuração em rede é peculiar ao ser humano. Ele se agrupa com seus semelhantes e estabelece relações de interesse, sejam elas de trabalho ou não, que se desenvolvem e se modificam conforme sua trajetória.

Ainda acerca das redes, Silva, Fialho e Saragoça (2013) destacam que significam uma estrutura de laços entre atores de determinado sistema social, que pode compreender indivíduos, organizações, setores ou estados-nação, e afirmam que os laços entre eles baseiam-se no diálogo, afeto, amizade, parentesco, autoridade, troca econômica, troca de informação ou qualquer outro meio que constitua o alicerce de uma relação.

As redes sociais estão presentes na Internet e representam um meio de comunicação, interação, entretenimento, conhecimento e negócios para os usuários. São definidas por Ellison, Steinfield e Lample (2007) como um espaço da Web que permite a seus usuários construir perfis públicos, articular suas redes de contatos e tornar visíveis essas conexões.

De forma complementar, Meira (2009) as considera como mecanismos que possibilitam a construção do imaginário coletivo e podem ser imprescindíveis para a criação e manutenção da sociedade em rede.

As redes sociais dizem respeito a pessoas e aos relacionamentos entre elas, e não somente à tecnologia. Sua essência é a comunicação, e as tecnologias são elementos que facilitam as interações (Gabriel, 2010). Algumas redes sociais de maior destaque no Brasil são o Facebook, Instagram e Twitter (Primo, 2007; Silva JR., 2012; Costa et al., 2014).

\section{As redes sociais, a Web 2.0 e os novos consumidores - os prosumers}

Minor e Mowen (2003) afirmam que a importância de compreender o consumidor é encontrada na definição do próprio marketing, que é a satisfação das necessidades e vontades dos clientes. Para os autores, um dos fatores de influência no comportamento do consumidor são as mudanças tecnológicas, pois constituem "uma fonte importante de ideias para novos produtos" (Minor; Mowen, 2003, p.27). Por isso, compreender a mudança tecnológica ocorrida nos últimos anos, a partir da Web 2.0, torna-se primordial.

Segundo O'Reilly (2005), o conceito de Web 2.0 nasceu em uma sessão de brainstorming entre Tim O'Reilly e profissionais da empresa Media Live International, com o objetivo de designar a mudança na forma como a web passou a ser utilizada e como os conteúdos são criados e disponibilizados por seu intermédio (O'Reilly, 2005). Já 
para Barreto (2011), o conceito Web 2.0 surgiu, primeiramente, em 2004, pelas mãos de Dale Dougherty, como meio de identificar a segunda geração de comunidades e serviços baseados numa plataforma web ou rede social, em que a diferença está na transição de uma era de produção para uma de colaboração, na qual o usuário passa a ser um ator atuante (Barreto, 2011). Amaral (2012) argumenta que na Web 2.0 o conteúdo é o novo suporte dos laços sociais, pois estes estão baseados em redes sociais, estruturadas para manter a interação. Assim surgem os prosumers, que desafiam os papéis tradicionais de consumidores e produtores, tornando o usuário produtor e consumidor simultaneamente.

Prosumer, em inglês, é a união da palavra producer (produtor) e consumer (consumidor). Significa que o consumidor é ao mesmo tempo produtor e consumidor de conteúdo. O termo prosumer foi utilizado pela primeira vez pelo futurólogo Alvin Tofler, em seu livro "A Terceira Onda", de 1980 (Troye; Xie, 2007; Fonseca et al., 2008; Islas-Carmona, 2008). Contudo, Islas-Carmona (2008) destaca que o conceito do termo foi utilizado inicialmente por Marshall McLuhan e Barrington Nevitt, no livro "Take Today", de 1972, no qual afirmavam que a tecnologia permitiria ao consumidor assumir o papel de produtor.

Os prosumers apresentam algumas características específicas, dentre as quais o fato de dominarem a tecnologia e a utilizarem para comunicar e fazer escolha, estarem sempre conectados e serem capazes de influenciar quem os rodeia. Além disso, os prosumers buscam valores nas marcas que consomem. Troye eXie (2007) afirmam que a compreensão do comportamento dos prosumers é fundamental na criação de ideias úteis e no entendimento das tendências de comportamento do consumidor, pois são consumidores proativos e dinâmicos, que compartilham seus pontos de vista e estão na vanguarda da tecnologia. Fonseca et al. (2008) destacam a evidente importância dos prosumers como influenciadores do mercado e da produção até o consumo. Tapscott (2010) afirma que o momento atual é de compartilhamento e ascensão das redes de influência; por isso, entender os prosumers é primordial para as empresas, pois o impacto que eles causam no mercado de consumo não está somente no poder de compra, mas no da influência. Existe o desejo de viver experiências e interagir com as empresas, os prosumers querem ser envolvidos na inovação dos produtos e estão se preparando para que as experiências ocorram (Tapscott, 2010).

\section{Inteligência Competitiva}

A forma sustentável de crescimento de uma organização incide sobro o conhecimento do mercado de atuação, dos concorrentes, dos clientes e dos consumidores. Assim, a informação, base do processo de Inteligência Competitiva, pode criar significado e construir esse conhecimento, auxiliando as organizações no planejamento estratégico e no processo de tomada de decisão (Queyras; Quoniam, 2006).

Para esclarecer o que é Inteligência Competitiva (IC), alguns conceitos se destacam, dentre os quais o de Fuld (1995), que define IC como informação analisada, que ajuda na tomada de decisão tática e estratégica. Para Miller (2002), o processo de inteligência está baseado na compreensão de que os gestores necessitam estar informados sobre diversas questões relacionadas ao negócio, de maneira formal e sistemática, gerando informações relacionadas a acontecimentos futuros que proporcionam vantagens sobre os concorrentes. A Inteligência Competitiva, para Tarapanoff (2006, p.26), é definida como "um processo de aprendizado motivado pela competição, fundado sobre a informação, permitindo esta última a otimização da estratégia corporativa em curto e em longo prazo".

Pode-se inferir que, a partir das definições de Inteligência Competitiva apresentadas, ela é um processo institucional, composto por planejamento, coleta, análise e disseminação de informações transformadas em inteligência, que amparam os gestores, com eficiência e eficácia, em seus processos decisórios.

As informações de inteligência estratégica são cada vez mais precisas, rápidas e confiáveis sobre fatos, tendências e relacionamentos presentes no ambiente competitivo em que as organizações atuam (Oliveira; 
Gonçalves; Paula, 2010). Para que o processo de inteligência competitiva alcance o resultado desejado, é fundamental desenvolver uma cultura interna que favoreça o compartilhamento das informações entre todos os membros da organização, além de promover o efetivo apoio da alta administração (Fuld, 1995; West, 2001; Miller, 2002; Bernhardt, 2003; Murphy, 2005; Rothberg; Erickson, 2005; Fleisher; Bensoussan, 2007).

\section{O processo de Inteligência Competitiva}

O processo de inteligência irá gerar informações embasadas em relação a acontecimentos futuros, para que os gestores possam tomar suas decisões com vantagem em relação a seus concorrentes, pois "os dados organizados transformam em informação; a informação quando analisada, transforma-se em inteligência" (Miller, 2002, p.35). A partir desse modelo se dá o processo ou ciclo de IC, compreendido por fases. Para Miller (2002), as fases são identificação das necessidades; coleta de informações relevantes; análise e síntese das informações; e disseminação da inteligência para os públicos de interesse. Prescott (2002) ressalta que existem vários modelos de Inteligência Competitiva, mas que a maioria é definida como Ciclo de Inteligência Competitiva, composta por cinco fases - identificação das necessidades; planejamento e direção; coleta; análise; e disseminação -, afirmando que nenhuma delas se sustenta sozinha, pois todas são necessárias e agregam valor umas às outras. Gomes e Braga (2004) denominam o processo de IC de Sistema de Inteligência Competitiva e afirmam que suas etapas são compreendidas em identificação das necessidades de informação; coleta; análise; disseminação; e avaliação.

A fase de identificação (1) compreende saber as reais necessidades dos tomadores de decisão e dos usuários, evitando a busca de dados desnecessários; o planejamento (2) envolve o levantamento das necessidades de inteligência e identificação dos envolvidos, bem como a elaboração de respostas às questões relacionadas aos tipos de processo de coleta e análise que serão utilizados e aos recursos disponíveis. Em seguida, na etapa de coleta (3) são selecionadas as fontes de dados e informações, que podem ser primárias ou secundárias, internas ou externas à empresa, formais ou informais. A fase de análise (4) é fundamental que seja realizada de forma proativa e criativa, por meio da prospecção, análise e comparação, mediante projeções e analogias para encontrar um significado de valor agregado. Por fim, as informações são transformadas em produtos de inteligência e podem ser disponibilizadas para implantação, disseminação e avaliação (5) (Miller, 2002; Gomes; Braga, 2004; Rodriguez y Rodriguez; Fontana, 2005).

Fuld (1995), Vaitsman (2001), Miller (2002), Nolan e Quinn (2002) propõem a inclusão de mais uma etapa no Ciclo de Inteligência Competitiva: o desenvolvimento de ações de contrainteligência (6), que objetivam prevenir ou neutralizar ações de inteligência realizadas pelos concorrentes.

Para se obter sucesso no processo de inteligência competitiva, é necessário saber que os resultados não são imediatos, pois ele necessita de tempo, recursos, pessoas, tecnologias e redes que facilitem os fluxos de informações (Fuld, 1995; Morais, 1999; Gomes; Braga, 2004).

\section{A importância das fontes de informação em Inteligência Competitiva}

As fontes de informações e os dados para análise são identificados na terceira etapa do Ciclo de Intenligência Competitiva (IC), a Coleta (Fuld, 1995; Miller, 2002; Nolan; Quinn, 2002; Gomes; Braga, 2004; Rodriguez y Rodriguez; Fontana, 2005), e são considerados fundamentais para o entendimento do ambiente organizacional (Porter, 1980; Choo, 1994; Fuld, 1995).

Aliar todos os tipos de fontes é fundamental para Fuld (1995), que afirma que somente assim é possível a resolução de problemas de inteligência. Segundo Calof (1999), saber gerenciar as fontes e validar a informação consultando várias fontes e métodos apropriados é imprescindível. Tomaél et al. (2001) corroboram Calof (1999) e 
propõem a verificação das fontes, destacando que a Internet possibilitou uma ampliação na tipologia de fontes de informações, algumas ainda não convencionais e não caracterizadas, sendo necessário o seu reconhecimento por meio da literatura.

Tomaél et al. (2001) apresentam os seguintes critérios de qualidade que devem ser observados, em relação à Internet, como, por exemplo, (a) informações cadastrais: dados detalhados do responsável pelo site, de forma a identificá-lo plenamente com o nome, endereço eletrônico para páginas Web, e-mail; (b) consistência das informações: detalhamento e robustez das informações que fornecem; (c) confiabilidade das informações: investiga a autoridade ou responsabilidade do produtor da fonte; (d) adequação da fonte: tipo de linguagem utilizada e coerência com os objetivos propostos; (e) links: internos e externos, observar se esses recursos complementam as informações e se são constantemente revisados; (f) facilidade de uso: facilidade para explorar/navegar no documento; (g) layout da fonte: mídias utilizadas; (h) restrições percebidas: situações que ocorrem durante o acesso e que podem restringir ou desestimular o uso de uma fonte de informação; (i) suporte ao usuário: elementos que fornecem auxílio aos usuários e que são importantes no uso da fonte; (j) outras informações percebidas como informações em outro idioma.

Existem muitos estudos para a avaliação de sites, ressalta Kaushik (2012), porém os estudos de avaliação dos recursos da Internet, caracterizados como websites, blogs, bibliografias, módulos de treinamento, banco de dados, e-books, redes sociais e newsletters, são poucos ou inexistentes. A autora cita os estudos da University of British Columbia, que apresentam seis critérios para avaliação dos recursos da Internet: autor ou fonte; precisão; atualização; objetividade; abrangência e propósito. Menciona ainda os critérios da Biblioteca da University of Queensland, que utiliza os seguintes indicadores: autor; credibilidade/responsabilidade; data; tipo de informação; escopo; propósito; estilo de escrita; língua utilizada e bibliografia. Nas pesquisas citadas, não há referências de estudos para a verificação, validação ou análise das redes sociais como fontes de informações (Kaushik, 2012). No contexto da Inteligência Competitiva, Barbosa $(2005$, p.4) afirma que as fontes de informação são utilizadas como "filtro para coletar elementos a respeito de aspectos ou componentes específicos do ambiente empresarial". Amaral (2006) destaca a importância da escolha certa das fontes, pois dessa forma é possível garantir a qualidade da informação. Azevedo (2012) corrobora Amaral (2006) ao afirmar que a relevância das fontes e a qualidade da informação são primordiais, pois somente assim é possível ter uma informação de valor para a empresa.

\section{Inteligência Competitiva e as redes sociais}

As redes sociais estão repletas de informações privilegiadas ou únicas e adquirem papel importante nas transações econômicas. Para Alcará et al. (2006), elas constituem estratégias para a viabilização da Inteligência Competitiva devido ao fortalecimento da cultura informacional e do compartilhamento do conhecimento e da informação. Lager (2009) destaca que as empresas podem se tornar mais produtivas se utilizarem as redes sociais para receber ideias dos clientes e manter um diálogo com eles. Ainda afirma que é possível estabelecer a credibilidade, prospectar vendas, participar de comunidades, apoiar a equipe de vendas, construir uma boa reputação online e assegurar a satisfação do cliente, mantendo-o sempre próximo (Lager, 2009).

Explorar o potencial das redes sociais por meio da criação de valor na construção de relacionamentos; cuidar do conteúdo disponibilizado; integrar a estratégia das redes sociais às estratégias da organização, principalmente, de relacionamento; e estar apto a dar respostas rápidas aos clientes, para Dong-Hun (2010), é um diferencial para as organizações estarem à frente em seus mercados de atuação. Dey et al. (2011) relatam que com base no monitoramento das redes sociais é possível identificar inúmeras informações: relacionadas à empresa, como contratação de novos funcionários; sobre a estratégia dos concorrentes, como o lançamento de produtos, registro de patentes e investimentos; sobre a opinião e o sentimento dos consumidores, por meio de informações de como eles comparam os produtos e até ideias para inovação; sobre os eventos dos concorrentes, promoções ou ofertas; 
e, por fim, acerca de acontecimentos gerais, mercado, economia ou outros assuntos, que podem ser globais, mas ter forte apelo local. Para os autores, a dificuldade encontrada ao lidar com a Internet e as mídias sociais ocorre em relação aos conteúdos que não interessam, entre eles elementos de linguagem difíceis de ser interpretados, propagandas, menus e pop-ups.

Vidigal (2011, p.45) afirma que as empresas se "diferenciam pelo que sabem" e que as funções internas das organizações, cada vez mais, são voltadas para o desenvolvimento de produtos e processos, sendo que as que possuem mais chance de prosperidade são as funções que unem o maior potencial de obtenção de vantagem competitiva e formas efetivas de sustentá-las. Para o autor, a utilização da Internet, mais especificamente das redes sociais, por parte das empresas pode gerar uma ligação inteligente de relacionamento e visibilidade perante os consumidores, e que é possível, estrategicamente, retirar a informação e utilizá-la de forma a obter vantagens.

\section{Procedimentos metodológicos}

A metodologia aplicada para a realização da pesquisa que deu origem ao presente artigo pode ser classificada como qualitativa, exploratória e descritiva (Gil, 2008; Vieira; Tibola, 2005). Utilizou-se o estudo de casos múltiplos, baseado em acessibilidade, com cinco empresas brasileiras, de portes distintos, que realizam a atividade de Inteligência Competitiva, no segmento de educação, tecnologia, publicidade e consultoria, denominadas como: Soluções Digitais (Empresa A); Educação e Editora (Empresa B); Mediação Social e Sustentabilidade (Empresa C); Publicidade e Propaganda (Empresa D); Consultoria em Inteligência de Mercado (Empresa E).

Foram realizadas entrevistas em profundidade com as pessoas responsáveis pela atividade de IC em cada uma das organizações. A pesquisa foi realizada nos meses de outubro e novembro de 2015, por meio de entrevistas semiestruturadas, analisadas em relação ao seu conteúdo (Bardin, 2002), estruturadas com o auxílio do software Atlas.ti, que permitiu atribuir importância e relações entre os fatores pesquisados.

No estudo foram avaliados: (a) o processo de coleta de informações das empresas, principalmente tendo as redes sociais como fontes de informação em Inteligência Competitiva; (b) os principais fatores pesquisados nas redes sociais que subsidiam a empresa pesquisada no processo de tomada de decisão, principalmente no desenvolvimento de produtos e serviços. Ao final do estudo, foi proposto um modelo analítico-conceitual para a verificação das redes sociais como fontes de informação na fase de coleta do processo de Inteligência Competitiva de empresas.

O modelo consiste em responder a questionamentos simples, de maneira formal, em seis etapas, relacionadas às informações disponibilizadas nas redes sociais, comentários, críticas ou sugestões de usuários diversos, pertinentes à empresa, a seus produtos e serviços, aos concorrentes e ao mercado, de forma que seja possível uma triagem das fontes, dados e das informações realmente relevantes para o processo de Inteligência Competitiva (Figura 1).

As questões são apresentadas em uma ficha de coleta (Quadro 1), adaptada de Janissek-Muniz, Lesca e Freitas (2006), a fim de facilitar a identificação e a utilização da informação por parte dos analistas das informações ou dos responsáveis pelas decisões.

\section{Resultados}

Em relação ao primeiro objetivo - análise do processo de Inteligência Competitiva e das Redes Sociais como fontes de coleta de informações -, foi possível identificar que a Empresa A possui uma área exclusiva para inteligência; a Empresa B está vinculada ao departamento de Marketing e Comercial; a Empresa E é toda voltada 


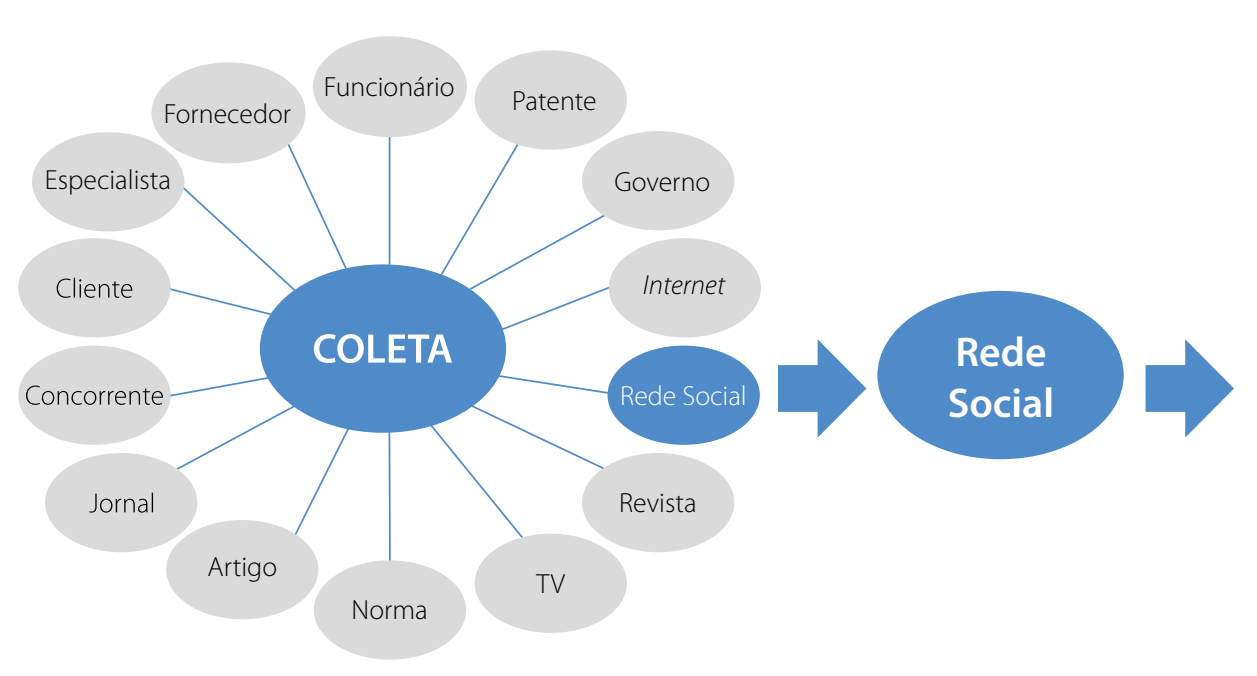

1. Identificar o usuário

2. Identificar a Rede Social

3. Categorizar o relacionamento

4. Relacionar a informação

5. Relatar tipo da informação

6. Identificar nível repercussão

Figura 1. Modelo proposto para verificação das RS como fonte em Inteligência Competitiva.

Fonte: Elaborado por Marques (2015), com base nos estudos de Tomaél et al. (2001) e Kaushik (2012).

para a IC; e nas empresas C e D o processo de IC é compartilhado entre os setores. Na Empresa D, em projetos específicos é designada uma equipe exclusiva para o trabalho. Em todas as entrevistas foi possível identificar que as atividades de IC são compartilhadas entre todos os envolvidos e a grande maioria delas é subordinada à direção da empresa.

Em relação aos objetivos de IC nas empresas, eles se mostraram diversos, de acordo com a respectiva área de atuação. A Empresa A tem o foco em IC Setorial e IC Territorial; a Empresa B, na melhoria e evolução dos produtos ofertados e no desenvolvimento de novas funcionalidades. Já as Empresas C, D e E traçam os objetivos baseados em projetos ou demandas específicas, de acordo com o cliente.

Quanto à infraestrutura e à equipe envolvida em IC, as empresas contam com profissionais de diversas áreas do conhecimento e a maioria delas não dispõe de softwares de mercado para o desenvolvimento do trabalho e, sim, de plataformas internas desenvolvidas para o processo.

No que diz respeito aos demandantes dos projetos em IC, foi possível verificar nas Empresas B, C e D que eles são internos, como participantes da direção ou gerentes. Já na Empresa A, os clientes externos são os principais demandantes, enquanto na Empresa E as solicitações partem dos dois lados, ou seja, tanto da direção quanto dos clientes externos, de acordo com o projeto ou serviço solicitado ou em andamento.

As técnicas de análise das informações no processo de IC compreendem: coleta de dados, organização, análise e desenvolvimento de relatórios (Empresa A); Swot (Strenghts, Weaknesses, Opportunities, Threats) e Indicadores Ethos (Empresa C), que destaca também a importância da percepção dos analistas, cuja experiência é, muitas vezes, decisória na análise e tomada de decisão; softwares de pesquisa como o Ibope, Instituto Verificador de Comunicação (IVC), IPSOS Marplan e pesquisas fornecidas pelo Conselho Executivo das Normas-Padrão (CENP) (Empresa D). A técnica de análise da Empresa E não foi relatada.

A análise das informações coletadas é uma das etapas do Ciclo de IC, sendo uma parte fundamental do processo que deve ser realizada de forma proativa, com o auxílio de softwares, técnicas e especialistas, que realizem comparações, projeções e analogias, conferindo, assim, significado às informações, para que elas se transformem em produtos de inteligência (Herring, 1997; Calof, 1999; Miller, 2002; Prescott, 2002; Gomes; Braga, 2004; Fuld, 2010). 
Quadro 1. Ficha de coleta de informações nas Redes Sociais.

Ficha de Coleta: Informações nas Redes Sociais

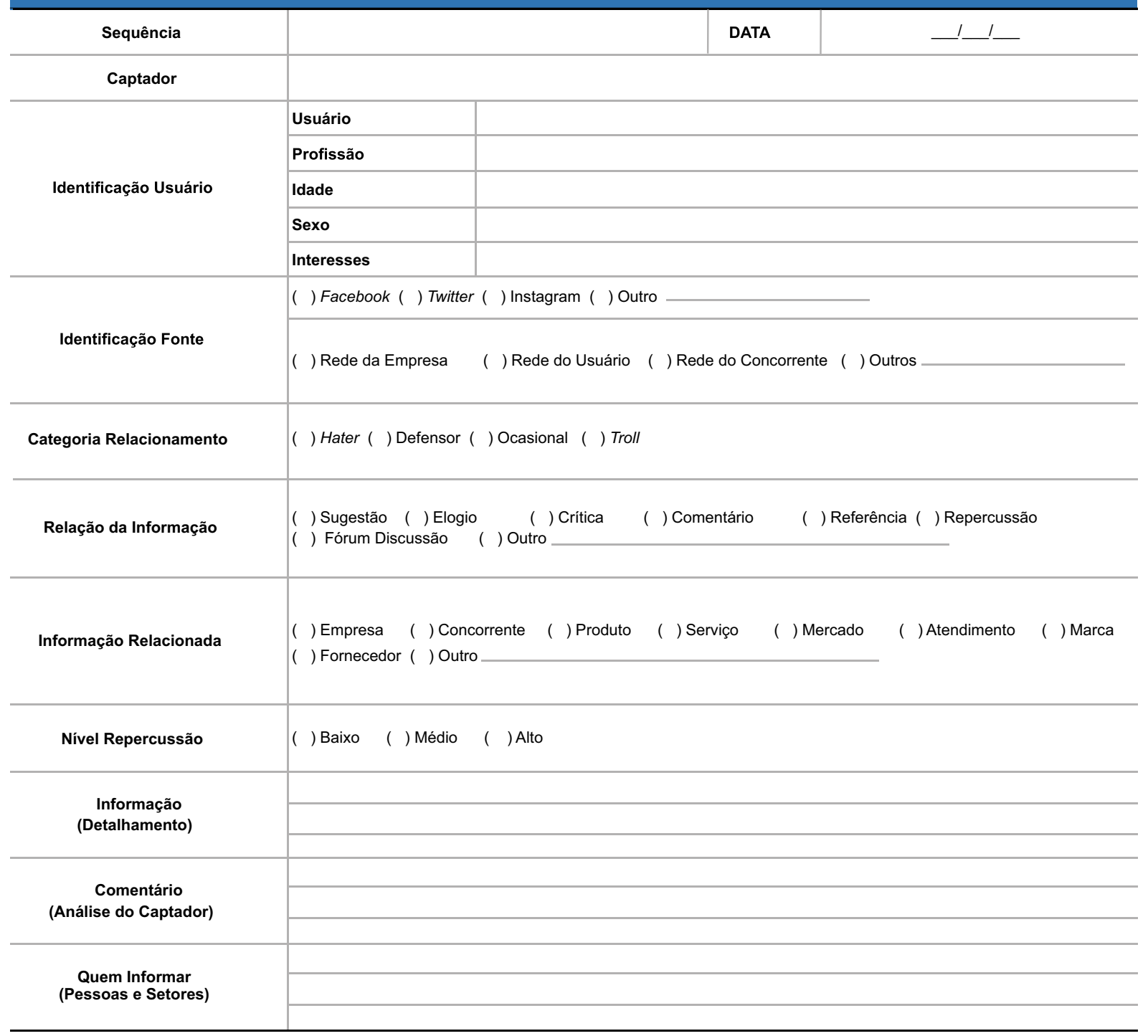

Fonte: Adaptado por Marques (2015), a partir de Janissek-Muniz, Lesca e Freitas (2006).

Pode-se inferir, com base na literatura, que as Empresas A e C realizam a técnica de análise não somente dos softwares, mas também a partir da visão crítica dos especialistas. Em relação às principais fontes de informação de Inteligência Competitiva utilizadas pelas empresas, percebe-se que todas essas, sem exceção, utilizam jornais, revistas, artigos científicos e periódicos. As redes sociais são utilizadas por todas as empresas, com exceção da Empresa E, que, apesar de não utilizá-las como fonte, destaca sua importância e a necessidade de sua verificação e validação.

A pesquisa indicou que todas as empresas pesquisadas possuem portais próprios na Internet e se relacionam com seus clientes e consumidores por meio deles. Com exceção da Empresa A, todas estão presentes nas redes 
sociais e atuam de forma interativa com os prosumers. No que diz respeito à forma como as empresas relatam realizar o processo de coleta de informações nas redes sociais, a Empresa A utiliza especialistas dos setores e materiais de referência da área; a Empresa B possui uma equipe formada por três pessoas para monitoramento da rede, e os demais profissionais da empresa, devido ao engajamento, também reportam casos específicos ou informações pertinentes, especialmente em suas localidades; a Empresa C consulta blogs e facebook de pessoas de referência, mas não relatou como o processo é realizado; a Empresa D relatou que não há um processo definido para a coleta, mas que são avaliados o perfil do usuário e a linguagem utilizada; a Empresa E não efetua diretamente a coleta de informações na rede, mas contrata pesquisas que são realizadas na Internet.

Em relação ao segundo objetivo - principais fatores pesquisados nas redes sociais que subsidiam as empresas pesquisadas no processo de tomada de decisão, especialmente no desenvolvimento de produtos e serviços -, foi possível constatar que a Empresa A é a única que pesquisa pelo menos 14 tipos de informações diferentes nas redes sociais, sobre fornecedores, linhas de financiamento e fomento, dentre outras. A Empresa E não consulta as redes para obter informações, porém informou que eventualmente as verifica para acompanhamento.

A fase da coleta é primordial em IC, e saber identificar quais os tipos de informação pesquisar é parte dessa fase. Nesse sentido, Tomaél, Alcará e Di Chiara (2005) destacam que o conhecimento precisa ser transformado e que o compartilhamento das informações nas redes sociais, que permeiam as relações dos indivíduos, é a inovação necessária para as organizações serem exitosas. Ainda, de acordo a literatura, é fundamental que as empresas, para alcançarem os resultados almejados, desenvolvam uma cultura que possibilite o compartilhamento das informações com toda a sua equipe (Fuld, 1995; West, 2001; Miller, 2002).

Sobre a influência dos prosumers em relação ao desenvolvimento de produtos e serviços da empresa, identificou-se que, na Empresa A, estes são desenvolvidos diretamente com os consumidores, que participam por meio de pesquisas de satisfação, workshops, conversas com especialistas e negócios dos setores. Na Empresa B, os prosumers influenciam na evolução dos produtos já ofertados, enquanto nas Empresas C e D, é avaliado caso a caso, pois os trabalhos são realizados por demanda. Já na Empresa E, a influência do prosumer é fundamental para o entendimento e dimensionamento de sua relação com a marca, produto e projetos que serão desenvolvidos.

Em relação à utilização das redes sociais para o entendimento dos prosumers, foi possível verificar que a Empresa A não as utiliza no setor de IC, pois existe um setor específico de comunicação digital, que realiza, entre outras mensurações, as métricas de análise; a Empresa B utiliza as redes para entender o prosumer, principalmente do Facebook, onde há uma forte interação entre a empresa e os usuários; a Empresa C não utiliza as redes para esse fim; já a Empresa D se empenhou na utilização das redes para melhor entender o consumidor e realizar uma análise de sua atitude; a Empresa E não utiliza as redes diretamente, mas faz análise de antropomarketing e pesquisa netnográfica.

Em relação ao fato de as informações coletadas nas redes sociais influenciarem no desenvolvimento de produtos ou serviços da empresa, foi possível verificar que a Empresa A atua principalmente na conquista de novos clientes e na identificação das necessidades; a Empresa B relata que as informações coletadas nas redes sociais auxiliam sim, porém confrontam essas informações com dados de pesquisas encomendadas; a Empresa C destaca que o maior produto oferecido pela empresa são os relatórios, que contêm as informações coletadas nas redes sociais, seja com uma informação, citação ou ilustração; a Empresa D utiliza as redes para ver a reação dos clientes, principalmente para promover o diálogo da interação; a Empresa E relata que as informações provenientes das redes sociais não influenciam em nada, pois não há verificação da rede e do que está postado, e considera que a variedade de comentários sem filtro, com erros diversos e xingamentos, não possibilita uma avaliação adequada.

Conforme destacado pela literatura, o momento atual é de compartilhamento e ascensão das redes de influência; por isso, entender os prosumers é primordial para as empresas, sendo fundamental entendê-los nas redes sociais (Tapscott, 2010). 
Em relação ao último objetivo - verificação das redes sociais como fontes de informação na fase da coleta no processo de inteligência competitiva -, as empresas pesquisadas foram questionadas sobre a importância de determinados critérios: a identificação do usuário; a identificação da rede social; a categorização do tipo de relacionamento do usuário com a empresa; a relação da informação disponibilizada e sobre o que ou quem ela trata; e o nível de repercussão da informação. Todas as empresas indicaram que esses são fatores essenciais para a verificação das redes como fonte de informação. Ao serem questionadas sobre outros critérios que consideram importantes para a verificação das redes sociais, percebeu-se que elas sugeriram fatores inerentes às suas áreas de atuação, como, por exemplo, abrangência e mobilização (Empresa C), critério que pode ser incluído no modelo proposto, de acordo com a necessidade da empresa. Apesar de considerarem fundamentais e importantes essas perguntas, as empresas pesquisadas não as realizam no momento de coleta da informação nas redes sociais; porém, sinalizaram que um roteiro com as perguntas seria de grande valia para a triagem e seleção das informações.

Assim, foi possível inferir que o modelo analítico-conceitual de verificação das redes sociais como fonte em Inteligência Competitiva proposto foi considerado importante e viável pelas empresas para a triagem das informações. Todos os itens foram apontados como norteadores para a verificação das informações disponibilizadas nas redes sociais. Esse resultado corrobora a literatura, que ressalta a necessidade de verificar a confiabilidade dessa nova fonte de informação. Alcará et al. (2006) enfatizam a utilização das redes sociais como uma fonte nova, que ainda precisa ser mais explorada pela literatura, pois os atores que nela se encontram fazem parte de nichos que são importantes para a organização. Para Azevedo (2012), a escolha certa das fontes de informação requer interesse e busca por informações confiáveis, adequadas para utilização, pois somente dessa forma é possível a correta coleta, seleção, análise e disseminação de informações relevantes.

\section{Conclusão}

Foi possível inferir que todas as empresas possuem um setor de Inteligência Competitiva bem estruturado, com profissionais de diversas áreas de atuação, formando uma equipe multidisciplinar de trabalho. Em relação aos modelos e técnicas de análise da informação, a ferramenta de Customer Relationship Management, cliente oculto, análise de concorrência, análise Swot e pesquisas diversas foram os mais citados. Para $60 \%$ das empresas os objetivos de Inteligência Competitiva estão relacionados a demandas ou projetos específicos dos clientes; $20 \%$ delas estão centradas na evolução dos produtos e desenvolvimento dos funcionários; e 20\% têm foco em Inteligência Competitiva setorial e territorial. Para $60 \%$ dos entrevistados, os demandantes de Inteligência Competitiva são os gestores da empresa; para 20\%, são o gestor e o cliente, de acordo com o projeto em andamento; e para os outros 20\%, apenas o cliente é o demandante de Inteligência Competitiva, também relacionado ao projeto em andamento ou solicitado. O relacionamento das empresas com os prosumers nas redes sociais é interativo para $80 \%$ dos entrevistados, enquanto apenas $20 \%$ estão ausentes das redes sociais e utilizam somente o site da empresa como canal informativo. Todas as empresas reconhecem a importância das redes sociais na atualidade e 80\% consideram as redes sociais como fontes de Inteligência Competitiva, um processo em desenvolvimento.

Em relação ao segundo objetivo, foi possível perceber que, para 80\% das empresas, as informações relacionadas ao ambiente econômico, legal, político e sociocultural são pesquisadas nas redes sociais, e o resultado coletado é confrontado com outras fontes de informação, entre elas a pesquisa de mercado. Para 20\% dos entrevistados, a consulta na rede social presta-se apenas para acompanhamento de alguma informação obtida por meio de outra fonte, como, por exemplo, verificar a repercussão de algum fato específico. Embora todas as empresas considerem que os prosumers influenciam diretamente no desenvolvimento e aprimoramento de seus produtos e serviços, somente $40 \%$ delas utilizam as redes sociais para o entendimento dos prosumers, e o fazem principalmente graças à interatividade do meio. Para $40 \%$ dos entrevistados, as informações coletadas nas redes 
sociais auxiliam a empresa no setor de marketing, enquanto para 20\% auxiliam em cursos e treinamentos para os funcionários, e para os outros 40\% não exercem nenhuma influência.

Em relação ao modelo analítico-conceitual de verificação das redes sociais como fonte de informações na fase de coleta de dados no Ciclo de Inteligência Competitiva, é interessante destacar que todos os itens apresentados foram considerados importantes e fundamentais. No entanto, nenhuma empresa realiza o questionamento proposto para verificar a importância das informações coletadas na rede: identificação do usuário e da rede social; categorização do tipo de relacionamento do usuário com a marca; tipo de informação disponibilizada; e repercussão da informação. O modelo proposto auxilia na verificação das informações disponibilizadas em diversas redes sociais, de forma ampliada, a partir de uma triagem simples, que tende a ajudar o gestor no confronto dos dados obtidos, aliado a outras fontes de informação que permitem prever cenários, aprimorar produtos, melhorar serviços e antecipar tendências, dentre outras possibilidades, proporcionando à empresa alcançar seus objetivos. Foram apontados outros itens de verificação, tais como, por exemplo, a abrangência da informação. Esse item ou outro, de acordo com a necessidade da empresa, pode ser acrescentado ao modelo, sem prejuízo, aprimorando ainda mais a verificação das redes sociais como fontes de informação. Assim, pode-se inferir que o modelo analítico-conceitual proposto foi considerado importante e viável para a triagem das informações mercadológicas, pelas empresas entrevistadas.

Baseando-se nos resultados da pesquisa e na literatura revisitada, identificou-se que todas as empresas pesquisadas monitoram as redes sociais como uma fonte de informação mercadológica e estratégica, ou seja, detectou-se que estas são geradoras de dados diversos que podem auxiliar não somente o desenvolvimento de produtos e serviços, como também outros processos decisórios e setores da empresa. Porém ressalta-se que é fundamental uma análise mais apurada, ou seja, uma verificação detalhada das redes para que as informações coletadas sejam realmente relevantes para os objetivos da empresa.

Entende-se que este estudo traz uma contribuição gerencial e acadêmica para o entendimento dos prosumers e das redes sociais como fontes de informação mercadológica. Ele aponta, ainda, que é possível, por meio de uma triagem simples e rápida, garantir a qualidade das informações dessas importantes fontes no processo de inteligência competitiva. Percebe-se que uma limitação do estudo está no número reduzido de unidades de análise, o que pode dificultar a generalização para outras populações. Como sugestão para estudos futuros indica-se a replicação deste estudo de forma a verificar o modelo proposto para outras empresas e, na medida do possível, com amostra probabilística. Outra sugestão é a realização de uma pesquisa netnográfica, que permita entender os prosumers em relação à empresa, à marca e aos produtos e serviços por ela ofertados.

\section{Colaboradores}

Todos os autores contribuíram na concepção e desenho do estudo, análise de dados e redação final.

\section{Referências}

Alcará, A. R. et al. As redes sociais como instrumento estratégico para a inteligência competitiva. Transinformação, v. 18, p. 143-153, 2006.

Amaral, I. Participação em rede: do utilizador ao "consumidor 2.0" e ao "prosumer". Revista Comunicação e Sociedade, n. 22, p. 131-147, 2012. Disponível em: <http://revistacomsoc.pt/ index.php/comsoc/article/view/1278/1220>. Acesso em: 22 jun. 2015.
Amaral, R. M. Desenvolvimento e aplicação de um método para o mapeamento de competências em inteligência competitiva. 2006. 207 f. Dissertação (Mestrado em Engenharia de Produção) - Universidade Federal de São Carlos, São Carlos, 2006.

Azevedo, A. W. Metodologia de identificação de fontes de coleta de informação: uma proposta de modelo para a cadeia produtiva de ouro e artefatos. Perspectivas em Gestão e Conhecimento, v. 2, p. 149-158, 2012. 
Barbosa, R. R. Uso de fontes de informação para a inteli-gência competitiva: um estudo da influência do porte das empresas sobre o comportamento informacional. In: Encontro Nacional de Pesquisa em Ciência da Informação - Enancib, 6., 2005, Florianópolis. Anais... Florianópolis: Ancib, 2005, p. 1-13. Disponível em <http://repositorios.questoesemrede.uff.br/ repositorios/bitstream/handle/123456789/260/GT4_Barbosa. pdf?sequence=1 >. Acesso em: 1 jul. 2015.

Bardin, L. Análise de conteúdo. Lisboa: Edições 70, 2002.

Barreto, A. M. Uma visão sobre a evolução da relação entre marcas e consumidores após a emergência da Web 2.0. Prisma.com, n. 15, 2011. Disponível em: <http://revistas.ua.pt/ index.php/prismacom/article/view/1088>. Acesso em: 1 jun. 2015.

Bernhardt, D. Competitive Intelligence: How to acquire and use corporate intelligence and counter-intelligence. London: Prentice Hall, 2003.

Calof, J. L. Teaching Cl: Opportunities and needs. Competitive Intelligence Magazine, v. 2, n. 4, p. 28-31, 1999.

Castells, M. A galáxia da internet: reflexões sobre a internet, os negócios e a sociedade. Rio de Janeiro: Jorge Zahar, 2003.

Choo, C. W. Perception and use of information sources in environmental scanning. Library and Information Science Research, v. 16, n. 1, p. 23-40, 1994.

Costa, M. et al. Curtir, comentar, compartilhar: o impacto da tipologia da postagem sobre a interação do cliente com a marca na rede social virtual. In: Encontro de Marketing, Anpad, 5., 2014, Gramado. Anais... Gramado: Anpad, 2014.

Dey, L. et al. Acquiring competitive intelligence from social media. In: Joint Workshop on Multilingual OCR and Analytics for Noisy Unstructured Text Data, 2011. Proceeding... Beijing, China: ACM, 2011. Available from: <http://delivery.acm. org/10.1145/ 2040000/2034621/a3-dey. pdf?ip=200. 228 . $81.53 \& i d=2034621 \&$ acc $=$ ACTIVE\%2OSERVICE\&key $=344 E$ 943C9DC262BB\%2E256698BBBBB5B74E\%2E4D4702B0C 3E38B35\%2E4D4702B0C3E38B35\&CFID $=998902576 \&$ CFTOKEN $=14290845 \& \_$acm__=1509020930_96613cb5 b216e f4adecaadb494293c70>. Cited: Oct. 25, 2017.

Dong-Hun, L. Growing popularity of social media and business strategy. SERI Quarterly, v. 3, n. 4, p. 112-117, 2010.

Ellison, N. B.; Steinfield, C.; Lample, C. The benefits of Facebook friends: Social capital and college student's use of online social network sites. Journal of Computer-Mediated Communications, v. 12, n. 4, 2007. Available from: <http://www.demonish.com/ cracker/1431083920_ff8fdd2c34/7.pdf>. Cited: Feb. 17, 2015.

Fleisher, C. S.; Bensoussan, B. E. Business and Competitive Analysis: Effective application of new and classic methods. [S.I.]: Financial Times Press, 2007.

Fonseca, M. A. et al. Tendências sobre as comunidades virtuais na perspectiva dos prosumers. In: Encontro de Marketing da Anpad, 3., 2008, Curitiba. Anais... Curitiba: Anpad, 2008.

Fuld, L. M. The Secret Language of Competitive Intelligence: How to see through and stay ahead of business disruptions, distortions, rumors and smoke screens. Indianápolis: DogEar Publishing, 2010.
Fuld, L. M. The new competitor intelligence: The complete resource for finding, analyzing and using information about your competitors. New York: John Wiley and Sons, 1995.

Gabriel, M. Marketing na era digital. São Paulo: Novatec, 2010.

Gil, A. C. Modos e técnicas de pesquisa social. 6. ed. São Paulo: Atlas, 2008.

Gomes, E.; Braga, F. Inteligência competitiva: como transformar informação em um negócio lucrativo. Rio de Janeiro: Campus, 2004.

Herring, J. P. What is intelligence analysis? Competitive Intelligence Magazine, v. 1, n. 2, p. 13-16, 1997.

Instituto Brasileiro de Opinião e Estatística. Pesquisa 2010. Disponível em: <http://www.ibope.com.br/>. Acesso en: 12 out. 2014.

Instituto Brasileiro de Opinião e Estatística. Pesquisa 2012. Disponível em: <http://www.ibope.com.br/>. Acesso em: 12 out. 2014.

Islas-Carmona, J. O. El prosumidor. El actor comunicativo de la sociedad de la ubicuidad. Palabra Clave, v. 11, n. 1, p. 29-39, 2008. Disponible en: <http://www.scielo.org.co/pdf/pacla/ v11n1/v11n01a03.pdf>. Acceso en: 22 jun. 2015.

Janissek-Muniz, R.; Lesca, H.; Freitas, H. Inteligência estratégica antecipativa e coletiva para tomada de decisão. Organizações em Contexto, v. 2, n. 4, p. 92-118, 2006.

Kaushik, A. Evaluation of internet resources: A review of selected literature. Brazilian Journal of Information Science, v. 6, n. 2, p. 65-88, 2012. Available from: <https://dialnet.unirioja. es/descarga/articulo/4329971/1.pdf>. Cited: Aug. 5, 2015.

Lager, M. Sales and social media: No one's social (Yet). CRM Magazine, p. 29-33, 2009. (Thematic issues: Who owns the social customer?).

Marques, L. K. S. O uso das redes sociais como fontes de inteligência competitiva: um estudo multicasos. 2015. 107 f. Dissertação (Mestrado em Administração) - Universidade Fumec, Belo Horizonte, 2015.

Meira, F. B. As encruzilhadas da autogestão: imaginário e simbólico nas empresas assumidas por trabalhadores. 2009. 358 f. Tese (Doutorado em Administração de Empresas) - Escola de Administração de Empresas, Fundação Getúlio Vargas, São Paulo, 2009. Disponível em: <http://bibliotecadigital. fgv.br/dspace/bitstream/handle/10438/4573/71050100714. pdf? sequence=1 . . Acesso em: 12 out. 2014.

Miller, J. P. O milênio da inteligência competitiva. Porto Alegre: Bookman, 2002.

Minor, M. S.; Mowen, J. C. Comportamento do consumidor. São Paulo: Prentice Hall, 2003.

Miranda, A. Sociedade da informação: globalização, identidade cultural e conteúdos. Ciência da Informação, v. 29, n. 2, p. 78-88, 2000. Disponível em: <http://www.scielo.br/scielo. php?script=sci_arttext\&pid=S0100-19652000000200010>. Acesso em: 1 jun. 2015.

Morais, E. F. C. Inteligência competitiva: estratégias para pequenas empresas. Brasília: CDT-NIC, 1999. 
Moya, E. Inteligencia en redes sociales. Barcelona: Editorial UOC, 2014. Disponible en: <https://books.google.com.br/

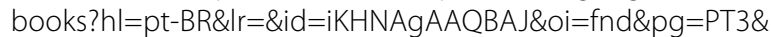
$\mathrm{dq}=$ moya $+2014+$ inteligencia\&ots $=7 \mathrm{sWUDcnJqM} \& \mathrm{sig}=\mathrm{n9D}$ Om3JJRV8p2sonZZWzXTNmz6c\#v=onepage \&q=moya\%20 2014\%20inteligencia\&f=false>. Acceso en: 22 jun. 2015.

Murphy, C. Competitive Intelligence: Gathering, analysing, and putting it to work. Aldershot: Gower Publishing Limited, 2005.

Nolan, J. A.; Quinn, J. F. Inteligência e segurança. In: Miller, J. P. O milênio da inteligência competitiva. Porto Alegre: Bookman, 2002. p. 229-249.

Oliveira, P. H.; Gonçalves, C. A.; Paula, E. M. Visão baseada em recursos da empresa, inteligência competitiva e balanced scorecard: em busca da vantagem competitiva sustentável. Revista Ciências da Administração, v. 2, n. 28, p. 60-82, 2010.

O'reilly, T. What is web 2.0. Design patterns and business models for the next generation of software. Design patterns and business models for the next generation of software. 2005. p. 1-5. Available from: <http://www.oreilly.com/pub/a/ web2/archive/what-is-web-20.html>. Cited: June 22, 2015

Porter, M. E. Competitive strategy: Techniques for analysing industries and competitors. New York: Free Press, 1980.

Prescott, J. E. Inteligência competitiva na prática: técnicas e práticas bem-sucedidas para conquistar mercados. Rio de Janeiro: Campus, 2002

Prescott, J. E.; Miller, S. H. Inteligência competitiva na prática: estudos de casos diretamente do campo de batalha. Rio de Janeiro: Campus, 2002.

Primo, A. O aspecto relacional das interações na Web 2.0. E-Compós, v. 9, p. 1-21, 2007. Disponível em: <http://www. ufrgs.br/limc/PDFs/web2.pdf>. Acesso em: 22 jun. 2015.

Queyras, J.; Quoniam, L. Inteligência Competitiva. In: Tarapanoff, K. (Org.). Inteligência, informação e conhecimento em corporações. Brasília: IBICT, 2006. p. 73-98.

Recuero, R. Redes sociais na internet. Porto Alegre: Sulina, 2009.

Rodrigues, L. C.; Riccardi, R. Inteligência competitiva nos negócios e organizações. Maringá: Unicorpore, 2007.

Rodriguez y Rodriguez, M. V.; Fontana, E. W. Inteligência Competitiva: nível de uso e influência nas receitas nos pequenos negócios exportadores. Revista Eletrônica de Administração, v. 11, n. 3, p. 1-26, 2005.
Rothberg, H. N.; Erickson, G. S. From knowledge to intelligence: Creating competitive advantage in the next economy. Woburn, MA: Elsevier, 2005.

Silva JR., J. A. Da fotografia expandida à fotografia desprendida: como o Instagram explica a crise da Kodak e vice-versa. In: Intercom, 35., 2012, Fortaleza. Anais... Fortaleza: Intercom, 2012.

Silva, C. A.; Fialho, J.; Saragoça, J. Análise de redes sociais e sociologia da acção. Pressupostos teórico-metodológicos. Revista Angolana de Sociologia, v. 11, p. 91-106, 2013. Disponível em: <http://ras.revues.org/361>. Acesso em: 15 nov. 2014.

Tapscott, D. A hora da geração digital: como os jovens que cresceram usando a internet estão mudando tudo, das empresas aos governos. Rio de Janeiro: Agir Negócios, 2010.

Tarapanoff, K. Inteligência organizacional e competitiva. Brasília: UNB, 2001.

Tarapanoff, K. (Org.). Inteligência, informação e conhecimento em corporações. Brasília: IBICT, 2006.

Tomaél, M.; Alcará, A.; Di Chiara, I. Das redes sociais à inovação. Ciência da Informação, v. 34, n. 2, p. 93-104, 2005. Disponível em: <http://www.scielo.br/pdf/ci/v34n2/28559.pdf/>. Acesso em: 17 dez. 2014

Tomaél, M. I. et al. Avaliação de fontes de informação na Internet: critérios de qualidade. Informação e Sociedade, v. 11, n. 2, p. 13-35, 2001. Disponível em: <http://periodicos.ufpb. br/ojs/index.php/ies/article/viewFile/293/216>. Acesso em: 30 ago. 2015.

Troye, S.; Xie, C. The active consumer: conceptual, methodological, and managerial challenges of prosumption. In: FIBE, 2007, Bergen. Conference... Bergen: NHH, 2007.

Vidigal, F. Inteligência competitiva: mapeamento de metodologias de uso estratégico da informação em organizações brasileiras. 2011. Tese (Doutorado em Ciência da Informação) - Escola de Ciência da Informação, Universidade Federal de Minas Gerais, Belo Horizonte, 2011.

Vieira, V. A.; Tibola, F. Pesquisa qualitativa em marketing e suas variações: trilhas para pesquisas futuras. Revista de Administração Contemporânea, v. 9, n. 2, p. 9-33, 2005.

West, C. Competitive intelligence. New York: Palgrave, 2001. 